\title{
Strain-Induced Enhancement of the Electron Energy Relaxation in Strongly Correlated Superconductors
}

\author{
C. Gadermaier, ${ }^{1 *}$ V. V. Kabanov, ${ }^{1}$ A. S. Alexandrov, ${ }^{1,2,3}$ L. Stojchevska, ${ }^{1}$ T. Mertelj, ${ }^{1}$ C. Manzoni, ${ }^{4}$ G. Cerullo, ${ }^{4}$ \\ N. D. Zhigadlo, ${ }^{5}$ J. Karpinski, ${ }^{5}$ Y. Q. Cai, ${ }^{6}$ X. Yao, ${ }^{6}$ Y. Toda, ${ }^{7}$ M. Oda, ${ }^{8}$ S. Sugai, ${ }^{9}$ and D. Mihailovic ${ }^{1}$ \\ ${ }^{1}$ Department of Complex Matter, Jozef Stefan Institute, Jamova 39, 1000 Ljubljana, Slovenia \\ ${ }^{2}$ Department of Physics, Loughborough University, Loughborough LE11 3TU, United Kingdom \\ ${ }^{3}$ Instituto de Física "Gleb Wataghin"/DFA, Universidade Estadual de Campinas-UNICAMP, \\ 13083-859 Campinas, Brazil \\ ${ }^{4}$ IFN-CNR, Dipartimento di Fisica, Politecnico di Milano, Piazza Leonardo da Vinci 32, 20133 Milano, Italy \\ ${ }^{5}$ Laboratory for Solid State Physics ETH Zurich, 8093 Zurich, Switzerland \\ ${ }^{6}$ Department of Physics, Shanghai Jiao Tong University, 800 Dongchuan Road, Shanghai 200240, China \\ ${ }^{7}$ Department of Applied Physics, Hokkaido University, Sapporo 060-8628, Japan \\ ${ }^{8}$ Department of Physics, Hokkaido University, Sapporo 060-0810, Japan \\ ${ }^{9}$ Department of Physics, Art and Science, Petroleum Institute, P.O. Box 2533, Abu Dhabi, United Arab \\ Emirates and Department of Physics, Faculty of Science, Nagoya University, \\ Furo-cho, Chikusa-ku, Nagoya 464-8602, Japan
}

(Received 25 December 2012; published 28 March 2014)

\begin{abstract}
We use femtosecond optical spectroscopy to systematically measure the primary energy relaxation rate $\Gamma_{1}$ of photoexcited carriers in cuprate and pnictide superconductors. We find that $\Gamma_{1}$ increases monotonically with increased negative strain in the crystallographic $a$ axis. Generally, the BardeenShockley deformation potential theorem and, specifically, pressure-induced Raman shifts reported in the literature suggest that increased negative strain enhances electron-phonon coupling, which implies that the observed direct correspondence between $a$ and $\Gamma_{1}$ is consistent with the canonical assignment of $\Gamma_{1}$ to the electron-phonon interaction. The well-known nonmonotonic dependence of the superconducting critical temperature $T_{c}$ on the $a$-axis strain is also reflected in a systematic dependence $T_{c}$ on $\Gamma_{1}$, with a distinct maximum at intermediate values $\left(\sim 16 \mathrm{ps}^{-1}\right.$ at room temperature). The empirical nonmonotonic systematic variation of $T_{c}$ with the strength of the electron-phonon interaction provides us with unique insight into the role of electron-phonon interaction in relation to the mechanism of high- $T_{c}$ superconductivity as a crossover phenomenon.
\end{abstract}

DOI: 10.1103/PhysRevX.4.011056

The deformation potential theorem [1] has been very successful in rationalizing and quantifying the strength of the electron-phonon interaction (EPI) in relation to charge carrier scattering and mobility in semiconductors [2,3], thus rapidly leading to an understanding of charge carrier dynamics and opening the way to modern semiconductor technology. Subsequently, it has been shown that the formalism is not limited to semiconductors, but can be extrapolated to both metals and insulators [4]. Strain on the structure caused by external pressure or doping may result in significant changes of the EPI, leading to changes in

\footnotetext{
*Corresponding author. christoph.gadermaier@ijs.si

Published by the American Physical Society under the terms of the Creative Commons Attribution 3.0 License. Further distribution of this work must maintain attribution to the author(s) and the published article's title, journal citation, and DOI.
}

Subject Areas: Condensed Matter Physics,

Superconductivity

functional properties over and above those caused by the changes of density of doped charges.

In both the cuprate and pnictide families of superconductors, the interatomic distance has been discussed as a quantitative parameter [5-7] in addition to the doping level $x$ that is systematically correlated with the superconducting critical temperature $T_{c}$. Although this was pointed out in cuprates long ago and is obviously of primary relevance to the superconducting mechanism, there is not even an elementary understanding of the origin of the peculiar nonmonotonic variation of $T_{c}$ on the lattice constant that is ubiquitously observed. Here, we present systematic measurements of electron energy relaxation in the normal state of different high- $T_{c}$ superconductors to show that in both cuprate and pnictide superconductors the primary electron energy relaxation rate $\Gamma_{1}$ is directly correlated with the length $a$ of the crystallographic $a$ axis, which in turn depends on the structure, the type of dopant atoms, and the doping level $x$. The dependence of the 
deformation potential on the lattice constant rationalizes how the EPI strength increases with increasing negative strain in the $a$ axis. Using established models that relate $\Gamma_{1}$ to the EPI, we make the connection between the EPI and $T_{c}$ for the first time. From the observed characteristic variation of $T_{c}$ on the EPI, we can reach important conclusions regarding the relevance of EPI to the superconducting mechanism in the context of strong correlations.

Following the proposal [8] and demonstration [9] of the use of femtosecond optical pump-probe spectroscopy to investigate the EPI in classical superconductors, we use an ultrashort laser pulse (the pump) to create a nonequilibrium electron distribution. The electron energy relaxation is probed by a second, weaker probe pulse, which detects the transient change in the reflectivity $\Delta R / R$. This method has been shown to yield a more reliable measure of the EPI strength [9-14] than the measurement of phonon linewidths by Raman or neutron scattering, which are often compromised by inhomogeneous broadening and probe only a limited selection of phonons.

Although the technique is experimentally well established, some precautions need to be taken to obtain quantitatively accurate data on $\Gamma_{1}$. In order to disentangle the various relaxation pathways in high- $T_{c}$ superconductors and determine their time constants $\tau_{i}=1 / \Gamma_{i}$, we use several different probe wavelengths. Since the $\tau_{i}$ depend on the sample temperature [see Eq. (1)], and additional processes appear in the presence of low-temperature order [15], we measure all samples at the same temperature$300 \mathrm{~K}$-well above the temperature where any lowtemperature ordering might take place. We avoid nonlinear, intensity-dependent relaxation processes and sample heating or degradation by keeping the pump pulse fluence below $20 \mu \mathrm{J} \mathrm{cm}^{-2}$. Most importantly, to provide the necessary time resolution and sensitivity, we built a system that gives tunable pump and probe wavelengths ranging from 500 to $700 \mathrm{~nm}$ with sub-30-fs overall time resolution. Sub-25-fs pump pulses with a repetition rate of $250 \mathrm{kHz}$ are provided by a noncollinear optical parametric amplifier tuned to a center wavelength of $535 \mathrm{~nm}$ (see the Supplemental Material [16] for a detailed description). Probe pulses are provided by a white light continuum generated in 2.5-mm-thick sapphire. Adequate time resolution is ensured via spectral filtering of the chirped probe pulses $[17,18]$. In several of the samples investigated here, this allowed the identification of previously unresolved relaxation processes.

The sample growth and doping is described elsewhere [19-24]. Samples are glued to a copper support using GE varnish. When needed, samples are cleaved to obtain a reflecting surface with low scattering. The reflectivity and the relative spectral weight of the signal components may vary across the surface, but the relaxation times do not. To eliminate the influence of the sample thickness, we used only samples much thicker than $100 \mu \mathrm{m}$, while our experiment probes a surface layer typically $\sim 100 \mathrm{~nm}$, depending on the material. The superconducting critical temperature $T_{c}$ (see Table I) was determined by measuring the temperature dependence of the ac magnetic susceptibility using a superconducting quantum interference device magnetometer.

A representative data set on the transient reflectivity of the selected compounds at room temperature is shown in Fig. 1. (The complete data are reported in the Supplemental Material [16].) To extract the relaxation times, we fit the data using single- or double-exponential decays and a Gaussian function for the instrumental response. (See the Supplemental Material [16] on the time resolution of the used setup and its implications for the accuracy of short $\tau_{1}$ values.) Oscillatory components are modeled using the same generation term as the main signal, leaving amplitude, frequency, phase, and damping time as the fit parameters. We measure all samples over a range of different wavelengths and in both orthogonal polarizations. Although we let all fit parameters vary freely between different wavelengths, for each sample, the best fits yield the same relaxation times within 10\%-20\%. From this observation, we conclude that at different wavelengths we are measuring the same relaxation processes but with different spectral weights.

From the fits, we find that the primary (fastest) relaxation rate $\Gamma_{1}$ ranges from 3 to $25 \mathrm{ps}^{-1}$ (i.e., relaxation time $\tau_{1}$ between 40 and $330 \mathrm{fs}$, Table I), independent of probe wavelength and sample orientation with respect to the probe polarization. For the cuprates, we also identify a slower relaxation component $\tau_{2}$. Some of the transient reflectivity signals show strong coherent phonon

TABLE I. Stoichiometries, superconducting critical temperatures, and electron-phonon relaxation times and rates of the investigated samples. For each compound family, we calculated error margins (standard deviation over the obtained fit parameters) for the sample with the highest number of measured time traces.

\begin{tabular}{lccc}
\hline \hline Sample & $T_{c}(K)$ & $\tau_{e-\mathrm{ph}}(\mathrm{fs})$ & $1 / \tau_{e \text {-ph }}\left(\mathrm{ps}^{-1}\right)$ \\
\hline $\mathrm{BaFe}_{2} \mathrm{As}_{2}$ & 0 & 300 & 3.3 \\
$\mathrm{Ba}\left(\mathrm{Fe}_{0.975} \mathrm{Co}_{0.025}\right)_{2} \mathrm{As}_{2}$ & 0 & 330 & 3.0 \\
$\mathrm{Ba}\left(\mathrm{Fe}_{0.949} \mathrm{Co}_{0.051}\right)_{2} \mathrm{As}_{2}$ & 20 & 320 & 3.1 \\
$\mathrm{Ba}\left(\mathrm{Fe}_{0.93} \mathrm{Co}_{0.07}\right)_{2} \mathrm{As}_{2}$ & 23 & 300 & 3.3 \\
$\mathrm{Ba}\left(\mathrm{Fe}_{0.89} \mathrm{Co}_{0.11}\right)_{2} \mathrm{As}_{2}$ & 10 & 300 & 3.3 \\
\hline $\mathrm{SmFeAsO}_{0.8} \mathrm{~F}_{0.2}$ & 49 & 190 & 5.3 \\
\hline $\mathrm{YBa}_{2} \mathrm{Cu}_{3} \mathrm{O}_{6.5}$ & 63 & 72 & 14 \\
$\mathrm{YBa}_{2} \mathrm{Cu}_{3} \mathrm{O}_{6.9}$ & 90 & 77 & 13 \\
\hline $\mathrm{HgBa}_{2} \mathrm{CuO}_{4.1}$ & 98 & 62 & 16 \\
\hline $\mathrm{Bi}_{2} \mathrm{Sr}_{2} \mathrm{CaCu}_{2} \mathrm{O}_{8.14}$ & 80 & 49 & 20 \\
\hline $\mathrm{La}_{1.9} \mathrm{Sr}_{0.1} \mathrm{CuO}_{4}$ & 30 & 42 & 24 \\
$\mathrm{La}_{1.85} \mathrm{Sr}_{0.15} \mathrm{CuO}_{4}$ & 38 & 45 & 22 \\
\hline \hline
\end{tabular}


oscillations [25], which are subtracted in the analysis. The $\tau_{1}$ values for $\mathrm{YBa}_{2} \mathrm{Cu}_{3} \mathrm{O}_{6+x}, \mathrm{Bi}_{2} \mathrm{Sr}_{2} \mathrm{CaCu}_{2} \mathrm{O}_{8.14}$, and the pnictides agree well with room-temperature data from the literature [10-13], while for $\mathrm{HgBaCa}_{2} \mathrm{CuO}_{4.1}$, they are resolved for the first time. For $\mathrm{La}_{2-x} \mathrm{Sr}_{x} \mathrm{CuO}_{4}$, our recent results obtained with much higher pump intensity [14] are confirmed by the present low-intensity data.

In Fig. 2, we plot the primary relaxation rate $\Gamma_{1}$ as a function of the crystallographic $a$ axis of pnictides and cuprates. All data points for cuprates, including differently doped samples, fit to a straight line, where $\Gamma_{1}$ decreases with increasing $a$. Defining the $a$-axis strain $\varepsilon_{a}=\Delta a / a_{0}$, with the equilibrium value [6] $a_{0}=3.94 \AA$ Á, this means that higher negative strain in the $\mathrm{Cu}-\mathrm{O}$ planes leads to faster

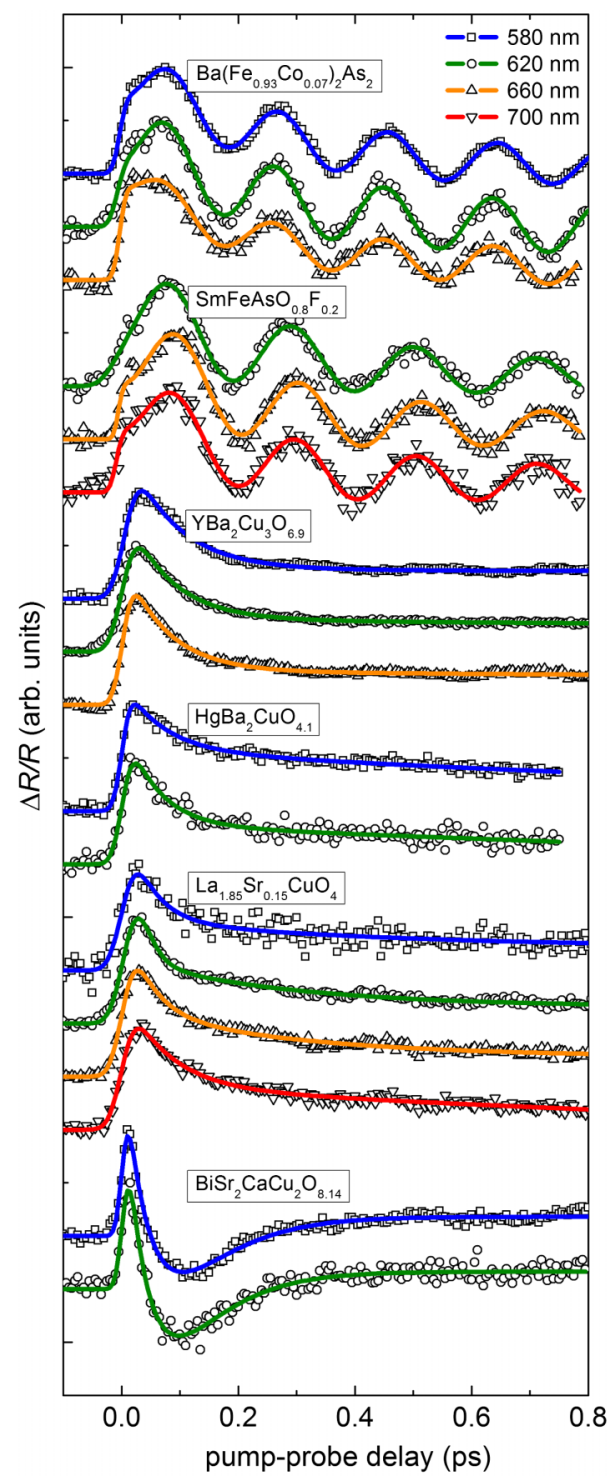

FIG. 1. Transient differential reflection at $295 \mathrm{~K}$ for different (near) optimally doped superconducting compounds. Thin solid lines are fits. Please refer to the Supplemental Material ([16], Figs. S8-S19) for different doping levels and sample orientations. electron energy relaxation. For a given cuprate, $a$ is not determined only by the type of compound, but also varies significantly with doping, by $0.1 \AA$ Á over the whole superconducting range of the phase diagram [5]. This translates into a doping-dependent relaxation rate, as our data for $\mathrm{YBa}_{2} \mathrm{Cu}_{3} \mathrm{O}_{6+x}$ and $\mathrm{La}_{2-x} \mathrm{Sr}_{x} \mathrm{CuO}_{4}$ at different doping levels confirm, and is in agreement with previous data [11] on $\mathrm{Bi}_{2} \mathrm{Sr}_{2} \mathrm{CaCu}_{2} \mathrm{O}_{8+x}$. On the other hand [26], $a$ in the pnictide $\mathrm{Ba}\left(\mathrm{Fe}_{1-\mathrm{x}} \mathrm{Co}_{\mathrm{x}}\right)_{2} \mathrm{Se}_{2}$ is nearly doping independent, changing by less than $0.002 \AA$. Remarkably, for all measured $\mathrm{Ba}\left(\mathrm{Fe}_{1-x} \mathrm{Co}_{x}\right)_{2} \mathrm{Se}_{2}$ samples, which cover a wide range of doping levels, the measured $\Gamma_{1}$ values show almost no variation. Hence, $\Gamma_{1}$ depends on $a$, but not on the doped carrier density.

Given the consensus in the literature in attributing the ultrafast response $\Gamma_{1}$ to the electron energy relaxation [10-14,27-29], we proceed to discuss the relaxation mechanism. The electron-phonon relaxation rate $1 / \tau_{e-\mathrm{ph}}$ can be related to the EPI strength expressed as the second moment $\lambda\left\langle\omega^{2}\right\rangle$ of the Eliashberg spectral function [8,30]:

$$
\frac{1}{\tau_{e-\mathrm{ph}}}=B \frac{\lambda\left\langle\omega^{2}\right\rangle}{T},
$$

where $T$ is the temperature and $B$ is a constant. Depending on whether one assumes the electron-electron relaxation to be much faster than the electron-phonon relaxation [8] or not [30], $T$ is either the electronic temperature $T_{e}$ or the lattice temperature $T_{L}$, respectively. In the present experiments, we use a very low laser excitation fluence, in which case $T_{e} \sim T_{L}$. The models above assume coupling between two baths: the hot electrons and the phonons whose coupling is characterized by the Eliashberg spectral function. One may also include more specific bosonic excitations in such models to emphasize a particular interaction [11,27-29]. However, this does not have significant bearing in the current discussion, since we are discussing only the dominant relaxation rate. The electron energy relaxes towards the bath that has the largest heat capacity; the lattice is the obvious candidate [31]. Recent microscopic calculations within the driven $t$ - $J$-Holstein model suggest that, under certain conditions, rapid energy transfer to spin degrees of freedom can occur [32], but considering the relaxation dynamics, only the relaxation of a highly excited charge carrier through Holstein phonons has been investigated thus far [33]. A detailed discussion of the assignment of $\Gamma_{1}$ to the EPI and the choice of the proportionality constant $B$ is given in the Supplemental Material [16].

The EPI strength is often described by a dimensionless parameter $\lambda$ (the zero-order moment of the Eliashberg spectral function) rather than $\lambda\left\langle\omega^{2}\right\rangle$. To calculate $\lambda$ from $\lambda\left\langle\omega^{2}\right\rangle$, one would need to know the complete $q$-and $\omega$ dependent Eliashberg spectral function. As a crude approximation, $\lambda\left\langle\omega^{2}\right\rangle$ is often simply divided by the square of an effective phonon frequency $\omega^{*}$. Commonly used 
values are $40 \mathrm{meV}$ for cuprates [21,29] and $25 \mathrm{meV}$ for pnictides [28]. Using these values, we estimate $\lambda \sim 0.3-0.5$ for cuprates and $\lambda \sim 0.2-0.3$ for pnictides. This estimate agrees well with $\lambda$ obtained from angle-resolved photoemission [34,35], neutron scattering [36-38], and tunneling [39] spectroscopy.

Generally, the effective electron-phonon coupling $\lambda$ is proportional to the square of the energy $E_{1}$, defined via the deformation potential $[1,40] \delta U=E_{1} \Delta$, which gives the energy shift of the conduction band edge as a function of the strain $\Delta$. For a small variation of $a$ (for the cuprates $\Delta a / a \sim 3 \%$ ), we may expand around the equilibrium value $a_{0}$ to first order:

$$
\begin{aligned}
\lambda *(a) & \approx \gamma\left[E_{1}\left(a_{0}\right)+\frac{\partial E_{1}}{\partial a}\left(a-a_{0}\right)\right]^{2} \\
& \approx \lambda *\left(a_{0}\right)+\tilde{\gamma} \frac{\partial E_{1}}{\partial a}\left(a-a_{0}\right) .
\end{aligned}
$$

The linear relation between $\lambda$ and $a$ above justifies the fit of $\Gamma_{1}(a)$ in Fig. 2. Using a simple estimate for $E_{1} \approx \hbar^{2} / 3 m a^{2}$, where $m$ is the electron mass, $\partial E_{1} / \partial a$ is negative, in agreement with the negative slope of the experimentally obtained $\Gamma_{1}(a)$.

In addition to the increase of $\lambda$ with decreasing $a$, the phonon frequencies observed by Raman spectroscopy also increase [41,42]. Both effects lead to an increase of the relaxation rate (see next paragraph). The observed blueshifts of the phonon bands by a few percent per picometer of contraction of $a$ are consistent in both sign and magnitude with the observed increase of $\Gamma_{1}$.

In both cuprates and pnictides, it has been proposed that $T_{c}$ is determined by two parameters: the doping level $x$ and the length $a$ of the crystallographic $a$ axis [5-7], or,

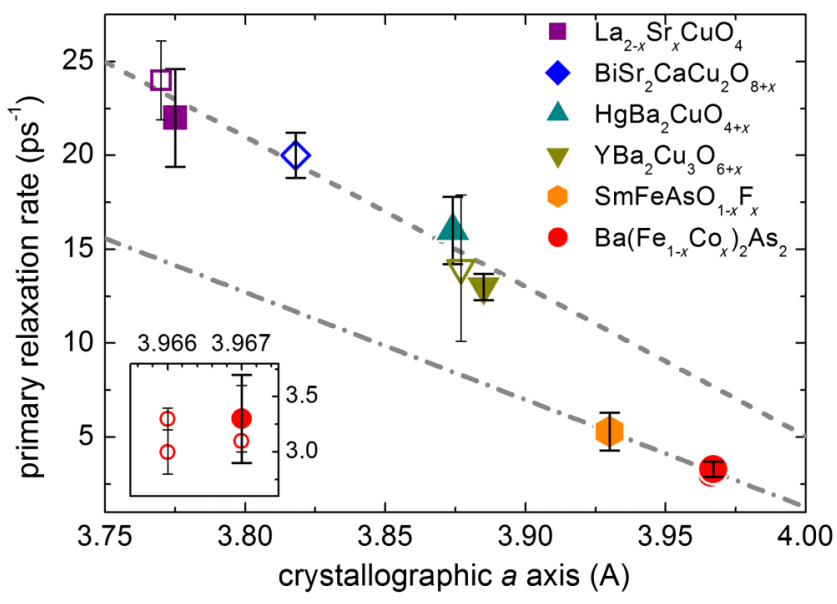

FIG. 2. Primary electron energy relaxation rate $\Gamma$ of pnictides and cuprates as a function of the $a$-axis length. Large symbols are at or close to optimal doping, open symbols at other doping levels. Dashed lines are linear fits. The inset is a zoom into the region of the five different $\mathrm{Ba}\left(\mathrm{Fe}_{0.975} \mathrm{Co}_{0.025}\right)_{2} \mathrm{As}_{2}$ samples, which have very similar a and $\Gamma$ values. equivalently, some related structural parameter, such as the $\mathrm{Cu}-\mathrm{O}$ in-plane bond length or the anion height in the pnictides. In the cuprates, superconductivity occurs when structure, doping-induced strain, or pressure-induced strain compresses $a$ well below its equilibrium value $[5,6]$ (in a cubic lattice) of $a=3.94 \AA$, with a maximum $T_{c}$ at $a=3.84$ Á.[Fig. 3(a)]. The direct correspondence between $a$ and $\Gamma_{1}$ shown in Fig. 2 suggests that, equivalently to $a, \Gamma_{1}$ can be used as a second parameter that determines $T_{c}$ [see Fig. 3(b)]. $T_{c}(R)$ systematically follows an arc with a distinct maximum around $K \sim 16 \mathrm{ps}^{-1}$. For the cuprates, $a$ and consequently $\Gamma_{1}$ depend on the doping levels, while for
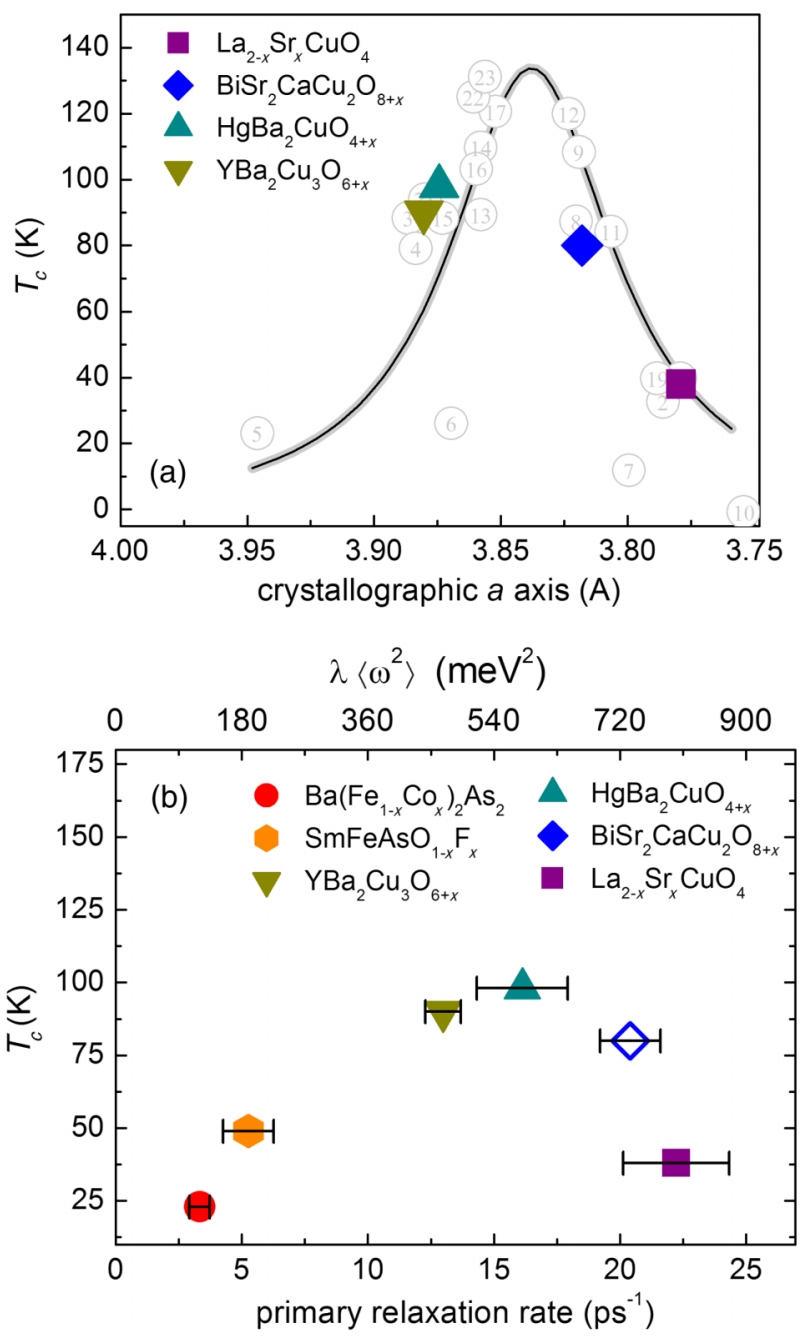

FIG. 3. (a) Critical temperature for cuprates as a function of the $a$ axis [2 times the in-plane $\mathrm{Cu}-\mathrm{O}$ distance as published in Ref. [5] (refer to the caption of Fig. 6 of Ref. [5] for the numbering of the compounds)]. Full symbols are our samples at or close to optimal doping (same symbol and color coding as Fig. 2), open circles are from Ref. [5], line is a fit to a Lorentzian as a guide to the eye. (b) The superconducting transition temperature $T_{c}$ as a function of the primary relaxation rate $\Gamma_{1}$. To plot the top axis, we used Eq. (1) with [30] $B=3 h / 2 \pi k_{B}$. Full symbols indicate (almost) optimally doped samples, open symbols other doping levels. 
$\mathrm{Ba}\left(\mathrm{Fe}_{1-x} \mathrm{Co}_{x}\right)_{2} \mathrm{Se}_{2}$ the two parameters are practically independent. The femtosecond pump-probe experiment, thus, directly yields a parameter $-\Gamma_{1}$-that is uniquely related to $T_{c}$.

Allen's proposition [8] that $\Gamma_{1}$ be proportional to the EPI and the Bardeen-Shockley deformation potential theorem [1] that relates the EPI to strain on the $a$ axis together suggest a correspondence between $a$ and the EPI, which we verified experimentally. Relating $T_{c}$ to the EPI rather than to $a$ can give us better insight into the applicability of different mechanisms of superconductivity. BCS theory, and by extension Eliashberg theory, predicts a monotonically increasing $T_{c}$ with increasing $\lambda$, which is contrary to the systematics we observe. On the other hand, in bare strongly correlated electron models, such as the Hubbard model and the $t-J$ model, the tunneling $t$ decreases with increasing distance, but the dependence of $T_{c}$ on $t$ is not clear. Until now, the dependence of other interactions, such as spin fluctuations, on the $a$ parameter has not been studied, but it is possible that these may have an-as yet undetermined-effect on $T_{c}$.

The observed nonmonotonic behavior strongly suggests a crossover phenomenon. Thus, competing interactions need to be considered, such as the crossover from weak coupling (BCS-like) to strong coupling [43] as a function of $\lambda$. An alternative approach includes strong correlations via the Coulomb interaction into an EPI model within the strong coupling approach [44]. In other words, the crossover behavior also highlights the necessity of the interplay of competing ground states [20] for achieving high $T_{c}$ 's. In any case, the EPI is clearly involved in a very unconventional way [45]. Indeed, the systematic, large, yet strongly unconventional isotope effect departing strongly from BCS behavior observed across the compounds corroborates this notion $[46,47]$. Although the present data and analysis presented in Fig. 3 do not identify the mechanism, they emphasize a role for unconventional electron-lattice effects and give a stringent verification criterion for any hopeful high- $T_{c}$ superconductivity theory $[48,49]$.

We thank D. Brida, F. Cilento, I. R. Fisher, P. Kusar, D. Polli, and L. Vidmar for stimulating discussions. This work was supported by the Slovenian Research Agency (ARRS) (Grants No. 430-66/2007-17, No. BI-CN/07-09003, and No. BI-IT/11-13-001), ROBOCON 2011-2012, the Royal Society (Grant No. JP090316), and the European Commission [Grant No. ERG-230975 and the European Community Access to Research Infrastructure Action, Contract No. RII3-CT-2003-506350 (Centre for Ultrafast Science and Biomedical Optics, LASERLAB-EUROPE)].

[1] J. Bardeen and W. Shockley, Deformation Potentials and Mobilities in Non-Polar Crystals, Phys. Rev. 80, 72 (1950).
[2] D. L. Rode, Electron Mobility in Direct-Gap Polar Semiconductors, Phys. Rev. B 2, 1012 (1970).

[3] M. V. Fischetti and S. E. Laux, Band Structure, Deformation Potentials, and Carrier Mobility in Strained Si, Ge, and SiGe Alloys, J. Appl. Phys. 80, 2234 (1996).

[4] R. Resta, Deformation-Potential Theorem in Metals and in Dielectrics, Phys. Rev. B 44, 11035 (1991).

[5] C. N. R. Rao and A. K. Ganguli, Structure-Property Relationships in Superconducting Cuprates, Chem. Soc. Rev. 24, 1 (1995).

[6] S. Agrestini, N. L. Saini, G. Bianconi, and A. Bianconi, The strain of $\mathrm{CuO}_{2}$ Lattice: The Second Variable for the Phase Diagram of Cuprate Perovskites, J. Phys. A 36, 9133 (2003).

[7] K. Horigane, H. Hiraka, and K. Ohoyama, Relationship between Structure and Superconductivity in $\mathrm{FeSe}_{1-x} \mathrm{Te}_{x}$, J. Phys. Soc. Jpn. 78, 074718 (2009).

[8] P. B. Allen, Theory of Thermal Relaxation of Electrons in Metals, Phys. Rev. Lett. 59, 1460 (1987).

[9] S. D. Brorson, A. Kazeroonian, J. S. Moodera, D. W. Face, T. K. Cheng, E. P. Ippen, M.S. Dresselhaus, and G. Dresselhaus, Femtosecond Room-Temperature Measurement of the Electron-Phonon Coupling Constant in Metallic Superconductors, Phys. Rev. Lett. 64, 2172 (1990).

[10] S. V. Chekalin, V. M. Farztdinov, V. V. Golovlyov, V. S. Letokhov, Y.E. Lozovik, Y. A. Matveets, and A. G. Stepanov, Femtosecond Spectroscopy of $\mathrm{YBa}_{2} \mathrm{Cu}_{3} \mathrm{O}_{7-\delta}$ : Electron-Phonon-Interaction Measurement and EnergyGap Observation, Phys. Rev. Lett. 67, 3860 (1991).

[11] E. E. M. Chia et al. Doping Dependence of the ElectronPhonon and Electron-Spin Fluctuation Interactions in the High- $T_{c}$ Superconductor $\mathrm{Bi}_{2} \mathrm{Sr}_{2} \mathrm{CaCu}_{2} \mathrm{O}_{8+\delta}$, New J. Phys. 15, 103027 (2013).

[12] T. Mertelj, V. V. Kabanov, C. Gadermaier, N. D. Zhigadlo, S. Katrych, J. Karpinski, and D. Mihailovic, Distinct Pseudogap and Quasiparticle Relaxation Dynamics in the Superconducting State of Nearly Optimally Doped $\mathrm{SmFeAsO}_{0.8} \mathrm{~F}_{0.2}$ Single Crystals, Phys. Rev. Lett. 102, 117002 (2009).

[13] T. Mertelj, P. Kusar, V. V. Kabanov, L. Stojchevska, N. D. Zhigadlo, S. Katrych, Z. Bukowski, J. Karpinski, S. Weyeneth, and D. Mihailovic, Quasiparticle Relaxation Dynamics in Spin-Density-Wave and Superconducting $\mathrm{SmFeAsO}_{1-x} \mathrm{~F}_{x}$ Single Crystals, Phys. Rev. B 81, 224504 (2010).

[14] C. Gadermaier, A. S. Alexandrov, V. V. Kabanov, P. Kusar, T. Mertelj, X. Yao, C. Manzoni, D. Brida, G. Cerullo, and D. Mihailovic, Electron-Phonon Coupling in High-Temperature Cuprate Superconductors Determined from Electron Relaxation Rates, Phys. Rev. Lett. 105, 257001 (2010).

[15] V. V. Kabanov, J. Demsar, B. Podobnik, and D. Mihailovic, Quasiparticle Relaxation Dynamics in Superconductors with Different Gap Structures: Theory and Experiments on $\mathrm{YBa}_{2} \mathrm{Cu}_{3} \mathrm{O}_{7-\delta}$, Phys. Rev. B 59, 1497 (1999).

[16] See Supplemental Material at http://link.aps.org/ supplemental/10.1103/PhysRevX.4.011056 for detailed description of the experimental technique and set-up, detailed description of the fitting procedure and assessment of the error margins of the obtained time constants, discussion of the assignment of the relaxation processes, complete raw data.

[17] D. Polli, D. Brida, S. Mukamel, G. Lanzani, and G. Cerullo, Effective Temporal Resolution in Pump-Probe Spectroscopy with Strongly Chirped Pulses Phys. Rev. A 82, 053809 (2010). 
[18] M. Liebel and P. Kukura, Broad-Band Impulsive Vibrational Spectroscopy of Excited Electronic States in the Time Domain, J. Phys. Chem. Lett. 4, 1358 (2013).

[19] N. D. Zhigadlo, S. Katrych, Z. Bukowski, S. Weyeneth, R. Puzniak, and J. Karpinski, Single Crystals of Superconducting $\mathrm{SmFeAsO}_{1-x} \mathrm{~F}_{y}$ Grown at High Pressure, J. Phys. Condens. Matter 20, 342202 (2008).

[20] J.-H. Chu, J. G. Analytis, C. Kucharczyk, and I. R. Fisher, Determination of the Phase Diagram of the Electron-Doped Superconductor $\mathrm{Ba}\left(\mathrm{Fe}_{1-x} \mathrm{Co}_{x}\right)_{2} \mathrm{As}_{2}$, Phys. Rev. B 79, 014506 (2009).

[21] J. Hofer, J. Karpinski, M. Willemin, G. I. Meijer, E. M. Kopnin, R. Molinski, H. Schwer, C. Rossel, and H. Keller Doping Dependence of Superconducting Parameters in $\mathrm{HgBa}_{2} \mathrm{CuO}_{4+\delta}$ Single Crystals, Physica (Amsterdam) 297C, 103 (1998).

[22] S. Sugai, H. Suzuki, Y. Takayanagi, T. Hosokawa, and N. Hayamizu, Carrier-Density-Dependent Momentum Shift of the Coherent Peak and the LO Phonon Mode in p-Type High- $T_{c}$ Superconductors, Phys. Rev. B 68, 184504 (2003).

[23] H. Gao, C. Ren, L. Shan, Y. Wang, Y. Zhang, S. Zhao, X. Yao, and H. H. Wen, Reversible Magnetization and Critical Fluctuations in Systematically Doped $\mathrm{YBa}_{2} \mathrm{Cu}_{3} \mathrm{O}_{7-\delta}$ Single Crystals, Phys. Rev. B 74, 020505(R) (2006).

[24] Y. H. Liu, Y. Toda, K. Shimatake, N. Momono, M. Oda, and M. Ido, Direct Observation of the Coexistence of the Pseudogap and Superconducting Quasiparticles in $\mathrm{Bi}_{2} \mathrm{Sr}_{2} \mathrm{CaCu}_{2} \mathrm{O}_{8+y}$ by Time-Resolved Optical Spectroscopy, Phys. Rev. Lett. 101, 137003 (2008).

[25] H. Takahashi, Y. Kamihara, H. Koguchi, T. Atou, H. Hosono, I. Katayama, J. Takeda, M. Kitajima, and K. G. Nakamura Coherent Optical Phonons in the Iron Oxypnictide $\mathrm{SmFeAsO}_{1-x} \mathrm{~F}_{x}(x=0.075)$, J. Phys. Soc. Jpn. 80, 013707 (2011).

[26] S. Drotziger, P. Schweiss, K. Grube, T. Wolf, P. Adelmann, C. Meingast, and H. v. Löhneysen Pressure versus Concentration Tuning of the Superconductivity in $\mathrm{Ba}\left(\mathrm{Fe}_{1-x} \mathrm{Co}_{x}\right)_{2} \mathrm{As}_{2}$ J. Phys. Soc. Jpn. 79, 124705 (2010).

[27] S. Dal Conte et al., Disentangling the Electronic and Phononic Glue in a High- $T_{c}$ Superconductor, Science 335, 1600 (2012).

[28] B. Mansart, D. Boschetto, A. Savoia, F. Rullier-Albenque, F. Bouquet, E. Papalazarou, A. Forget, D. Colson, A. Rousse, and M. Marsi, Ultrafast Transient Response and ElectronPhonon Coupling in the Iron-Pnictide Superconductor $\mathrm{Ba}\left(\mathrm{Fe}_{1-x} \mathrm{Co}_{x}\right)_{2} \mathrm{As}_{2}$, Phys. Rev. B 82, 024513 (2010).

[29] L. Perfetti, P. A. Loukakos, M. Lisowski, U. Bovensiepen, H. Eisaki, and M. Wolf, Ultrafast Electron Relaxation in Superconducting $\mathrm{Bi}_{2} \mathrm{Sr}_{2} \mathrm{CaCu}_{2} \mathrm{O}_{8+\delta}$ by Time-Resolved Photoelectron Spectroscopy, Phys. Rev. Lett. 99, 197001 (2007).

[30] V. V. Kabanov and A. S. Alexandrov, Electron Relaxation in Metals: Theory and Exact Analytical Solutions, Phys. Rev. B 78, 174514 (2008).

[31] P. Kusar, V. V. Kabanov, S. Sugai, J. Demsar, T. Mertelj, and D. Mihailovic, Controlled Vaporization of the Superconducting Condensate in Cuprate Superconductors by Femtosecond Photoexcitation, Phys. Rev. Lett. 101, 227001 (2008).

[32] L. Vidmar, J. Bonca, T. Tohyama, and S. Maekawa, Quantum Dynamics of a Driven Correlated System Coupled to Phonons, Phys. Rev. Lett. 107, 246404 (2011).
[33] D. Golez, J. Bonca, L. Vidmar, and S. A. Trugman, Relaxation Dynamics of the Holstein Polaron, Phys. Rev. Lett. 109, 236402 (2012).

[34] A. Lanzara et al. Evidence for Ubiquitous Strong ElectronPhonon Coupling in High-Temperature Superconductors, Nature (London) 412, 510 (2001).

[35] T. P. Devereaux, T. Cuk, Z.-X. Shen, and N. Nagaosa, Anisotropic Electron-Phonon Interaction in the Cuprates, Phys. Rev. Lett. 93, 117004 (2004).

[36] D. Reznik, L. Pintschovius, M. Ito, S. Iikubo, M. Sato, H. Goka, M. Fujita, K. Yamada, G. D. Gu, and J. M. Tranquada Electron-Phonon Coupling Reflecting Dynamic Charge Inhomogeneity in Copper Oxide Superconductors, Nature (London) 440, 1170 (2006).

[37] W. Meevasana, N. J. C. Ingle, D. H. Lu, J. R. Shi, F. Baumberger, K. M. Shen, W. S. Lee, T. Cuk, H. Eisaki, T. P. Devereaux, N. Nagaosa, J. Zaanen, and Z. X Shen, Doping Dependence of the Coupling of Electrons to Bosonic Modes in the Single-Layer High-Temperature $\mathrm{Bi}_{2} \mathrm{Sr}_{2} \mathrm{CuO}_{6}$ Superconductor, Phys. Rev. Lett. 96, 157003 (2006).

[38] R. Mittal, Y. Su, S. Rols, T. Chatterji, S. L. Chaplot, H. Schober, M. Rotter, D. Johrendt, and T. Brueckel, Inelastic Neutron Scattering and Lattice-Dynamical Calculations of $\mathrm{BaFe}_{2} \mathrm{As}_{2}$, Phys. Rev. B 78, 104514 (2008).

[39] J. Lee et al. Interplay of Electron-Lattice Interactions and Superconductivity in $\mathrm{Bi}_{2} \mathrm{Sr}_{2} \mathrm{CaCu}_{2} \mathrm{O}_{8+\delta}$, Nature (London) 442, 546 (2006).

[40] A. I. Anselm, Introduction to the Semiconducting Theory (LAN, St. Petersburg, Russian Federation, 2008), p. 470; J. M. Ziman, Electrons and Phonons (Oxford University Press, Oxford, 1960).

[41] A. F. Goncharov, M. R. Muinov, T. G. Uvarova, and S. M. Stishov, Raman Scattering of $\mathrm{YBa}_{2} \mathrm{Cu}_{3} \mathrm{O}_{x}$, Single Crystals with Different Oxygen Content at High Pressures, JETP Lett. 54, 111 (1991).

[42] M. Osada, M. Kakihana, H. Arashi, M. Kall, and L. Borjesson, Phonon Raman Scattering of $\mathrm{Bi}_{2} \mathrm{Sr}_{2} \mathrm{CaCu}_{2} \mathrm{O}_{8+d}$ under Hydrostatic Pressure, Phys. Rev. B 59, 8447 (1999).

[43] A.S. Alexandrov, Bipolarons in Narrow-Band Crystals, Zh. Fi. Khim. 57, 273 (1983) [Russ. J. Phys. Chem. 57, 167 (1983)].

[44] D. Mihailovic, V. V. Kabanov, and K. A. Müller, The Attainable Superconducting $T_{c}$ in a Model of Phase Coherence by Percolating, Europhys. Lett. 57, 254 (2002).

[45] A. J. Leggett, What Do We Know About High $T_{c}$ ?, Nat. Phys. 2, 134 (2006).

[46] A. Bussmann-Holder and H. Keller, Isotope and Multiband Effects in Layered Superconductors, J. Phys. Condens. Matter 24, 233201 (2012).

[47] A. S. Alexandrov, Transition from Fermi Liquid to Charged Bose Liquid: A Possible Explanation of the Isotope Shift in High-T ${ }_{c}$ Oxides, Phys. Rev. B 46, 14932 (1992).

[48] S. Johnston, F. Vernay, B. Moritz, Z.-X. Shen, N. Nagaosa, J. Zaanen, and T.P. Devereaux Systematic Study of Electron-Phonon Coupling to Oxygen Modes across the Cuprates Phys. Rev. B 82, 064513 (2010).

[49] D. J. Scalapino, A Common Thread: The Pairing Interaction for Unconventional Superconductors, Rev. Mod. Phys. 84, 1383 (2012). 